Article

\title{
Holomorphic Approximation on Certain Weakly Pseudoconvex Domains in $\mathbb{C}^{n}$
}

\author{
Shaban Khidr 1,2 \\ 1 Department of Mathematics, Faculty of Science, University of Jeddah, Jeddah 21589, Saudi Arabia; \\ skhidr@uj.edu.sa \\ 2 Department of Mathematics, Faculty of Science, Beni-Suef University, Beni-Suef 62511, Egypt
}

Received: 11 September 2019; Accepted: 24 October 2019; Published: 3 November 2019

\begin{abstract}
The purpose of this paper is to study the Mergelyan approximation property in $L^{p}$ and $\mathcal{C}^{k}$-scales on certain weakly pseudoconvex domains of finite/infinite type in $\mathbb{C}^{n}$. At the heart of our results lies the solvability of the additive Cousin problem with bounds as well as estimates of the $\bar{\partial}$-equation in the corresponding topologies.
\end{abstract}

Keywords: Mergelyan property; weakly pseudoconvex domains; additive Cousin problem

\section{Introduction}

The problem of approximation by holomorphic functions in one or several complex variables has been a central theme in complex analysis and finds it applications in many branches of mathematical physics such as systems identification, signal processing, inverse problems for partial differential equations, and operator theory (cf. [1]).

Holomorphic approximation in one complex variable goes back to Mergelyan [2] who proved that if $K$ is a compact set in the complex plane and its complement in $\mathbb{C}$ has just one connected component, then every continuous function on $K$ which is holomorphic in the interior of $K$ can be approximated on $K$ by holomorphic functions in a neighborhood of $K$.

Analogous problems in several complex variables were first investigated independently by Henkin [3], Lieb [4], and Kerzman [5] for strictly pseudoconvex domains with sufficiently smooth boundaries in $\mathbb{C}^{n}$, and subsequently improved by Fornæss [6] for domains with $\mathcal{C}^{2}$-smooth boundaries.

In particular, Kerzman [5] proved that if $D$ is a strongly pseudoconvex domain with $\mathcal{C}^{4}$-boundary in $\mathbb{C}^{n}$ (or in a Stein manifold), $n \geq 2$, then every holomorphic function in $L^{p}(D)$ can be approximated on $\bar{D}$ by holomorphic functions on a neighborhood of $\bar{D}$ in the scale of $L^{p}$-norm, $1 \leq p \leq \infty$.

In the $\mathcal{C}^{k}$-topology, the problem was first handled by Ahern-Schneider [7] and Lieb-Range [8]. Other generalizations can be found in [9].

The key technical tools needed to prove the above results were the existence of a Stein neighborhood basis for $\bar{D}$ and the integral solution operators for $\bar{\partial}$ with estimates in those topologies.

For weakly pseudoconvex domains, Fornæss and Nagel [10] proved that if $D$ is a smoothly bounded pseudoconvex domain with real analytic boundary, then every function continuous on $\bar{D}$, holomorphic in $D$, and can be approximated uniformly on $\bar{D}$ by holomorphic functions on a neighborhood of $\bar{D}$.

Moreover, Cho proved in [11] that if $D$ is a smoothly bounded pseudoconvex domain of finite type in $\mathbb{C}^{2}$, then every holomorphic function in the $L^{p}$-Sobolev space $W^{s, p}(D), 1<p<\infty, s \geq 0$, can be approximated on $\bar{D}$ by holomorphic functions on a neighborhood of $\bar{D}$ in the $W^{s, p}(D)$-norm. In addition, he obtained the same result for the usual Lipschitz space. His key ingredient was the stability of the estimates for $\bar{\partial}$ in the $W^{s, 2}$-setting. 
Recently, Kim [12] extended those results obtained in [5] for certain convex ellipsoids of finite/infinite type in $\mathbb{C}^{2}$. His proof is based on $L^{p}$-bounds in the additive Cousin problem via solvability of $\bar{\partial}$ with $L^{p}$-estimates on such domains.

On the other hand, Diederich and Fornæss [13] gave an example of smoothly bounded pseudoconvex domain $D$ in $\mathbb{C}^{2}$ known as "worm domain" for which the Mergelyan property fails. Their example is based on the presence of a Levi-flat hypersurface in $\partial D$ having an annular leaf with infinitesimally nontrivial holonomy.

The main object of this paper is to prove the Mergelyan property on certain class of weakly pseudoconvex domains in $\mathbb{C}^{n}, n \geq 2$, in different topologies like $L^{p}$-topology or $\mathcal{C}^{k}$-topology on compact subsets, taking for granted the existence of $\bar{\partial}$-solving bounded solution operators with estimation in the corresponding norms.

\section{Results}

Before formulating our first result, we recall from [14] the following definition.

Definition 1. Let $D_{\phi}$ be a $\mathcal{C}^{2}$ weakly pseudoconvex type domain defined by

$$
D_{\phi}=\left\{z \in \mathbb{C}^{n} ;\left|z_{j}\right|<1, j=1, \ldots, n ; \sigma(z)=\sum_{j=1}^{n-1}\left|z_{j}\right|^{2}+\phi\left(\left|z_{n}\right|^{2}\right)-1<0\right\},
$$

where $\phi$ is a $\mathcal{C}^{2}$ real-valued function $\phi$ on $[0,1]$ such that

(a) $\phi(0)=0$ and $\phi(1)=1$;

(b) $\phi^{\prime}(t)>0 ; 0<t \leq 1$;

(c) $\phi^{\prime}(t)+t \phi^{\prime \prime}(t)>0 ; 0<t \leq 1$;

(d) there is $\tau \in(0,1)$ so that $\phi^{\prime \prime}(t)>0,0<t \leq \tau$.

We denote by $L_{0, q}^{p}\left(D_{\phi}\right)$ the Banach space of $(0, q)$-forms on $D_{\phi}$ with $L^{p}$-coefficients. The following $L^{p}$-existence theorem for $\bar{\partial}$ on such domain $D_{\phi}$ was proved in [14].

Theorem 1. Let $D_{\phi}$ be given as above and assume that $\int_{0}^{1}|\log \phi(x)| x^{-\frac{1}{2}} d x<\infty$. Let $f \in L_{0, q}^{1}\left(D_{\phi}\right), q \geq 1$. Then there exist integral kernels $K_{q}(\xi, z)$ such that the integral $\int_{D_{\phi}} f(\xi) \wedge K_{q-1}(\xi, z) d \mu(\xi)$ is absolutely convergent for almost all $z \in \overline{D_{\phi}}$ and defines linear solution operators $T_{q}: L_{0, q}^{1}\left(D_{\phi}\right) \rightarrow L_{0, q-1}^{1}\left(D_{\phi}\right)$ to the equation $\bar{\partial} T_{q} f=f$ in $D_{\phi}$ provided that $\bar{\partial} f=0$, where $d \mu(\xi)$ is the Lebesgue measure on $\mathbb{C}^{n}$. In addition, if $f \in L_{0, q}^{p}\left(D_{\phi}\right), 1 \leq p \leq \infty$, then for each $p$ there is a constant $c(p)>0$, independent of $f$, satisfying the estimates

$$
\left\|T_{q} f\right\|_{L_{0, q-1}^{p}\left(D_{\phi}\right)} \leq c(p)\|f\|_{L_{0, q}^{p}\left(D_{\phi}\right)} .
$$

If $f$ is $\mathcal{C}^{\infty}$ then also $T_{q} f$ is $\mathcal{C}^{\infty}$.

For $\alpha>0$, define $\phi_{\alpha}(t)=e \exp \left(-1 / t^{\alpha}\right), 0 \leq t \leq 1$. Then $\phi_{\alpha}$ satisfies all conditions (a)-(d). In this case the domain $D_{\phi_{\alpha}}$ is of infinite type and satisfying the conditions of Theorem 1 if and only if $\alpha<\frac{1}{2}$.

For a bounded domain $D$ in $\mathbb{C}^{n}$, we denote by $\mathcal{O}(D)$ the set of all holomorphic functions on $D$ and by $\mathcal{C}(D)$ the set of continuous functions on $D$. By $H^{p}(D)$ we denote the set of all holomorphic functions in $L^{p}(D), 1 \leq p<\infty$. Finally, $H^{\infty}(D)$ denotes the weak-star closure of the algebra of functions that are continuous on $\bar{D}$ and holomorphic in $D$.

Now we formulate our first result which concerns approximating by functions that are holomorphic in some neighborhood of $\overline{D_{\phi}}$ in $L^{p}$-spaces. 
Theorem 2. Let $D_{\phi}$ be as in Theorem 1. Then every function in $H^{p}\left(D_{\phi}\right)$ can be approximated in $L^{p}\left(D_{\phi}\right)-n o r m$ by functions in $\mathcal{O}\left(\overline{D_{\phi}}\right)$ for all $1 \leq p<\infty$. For $p=\infty$, there is a constant $C<\infty$, such that every $f$ in $H^{\infty}\left(D_{\phi}\right) \cap \mathcal{C}\left(\bar{D}_{\phi}\right)$ can be approximated by a sequence $f_{j}$ in $\mathcal{O}\left(\overline{D_{\phi}}\right)$ in $L^{p}\left(D_{\phi}\right)$-norm, $1 \leq p<\infty$, such that $\left\|f_{j}\right\|_{L^{\infty}\left(D_{\phi}\right)} \leq C\|f\|_{L^{\infty}\left(D_{\phi}\right)}$ for all $j \geq 1$. Moreover, if $f$ extends continuously to $\overline{D_{\phi}}$, then $f_{j} \rightarrow f$ uniformly on $\overline{D_{\phi}}$.

Remark 1. It worth remarking that:

(i) This theorem was very recently obtained in [12] for domains in $\mathbb{C}^{2}$ satisfying slightly general conditions than ours.

(ii) A simple example of a pseudoconvex domain for which this result fails is the Hartogs triangle $H=$ $\left\{\left(z_{1}, z_{2}\right) \in \mathbb{C}^{2}: 0<\left|z_{2}\right|<\left|z_{1}\right|<1\right\}$. The holomorphic function $f\left(z_{1}, z_{2}\right)=z_{2} / z_{1}$ on $H$ is bounded by one, and it cannot be approximated in any natural sense by holomorphic functions in neighborhoods of $\bar{H}$ since its restriction to horizontal slices $z_{2}=$ const has winding number -1 . Note that $\bar{H}$ is not a Stein compact. One can also see that it fails on the Diederich-Fornæss worm domain [13].

Let $D$ be the complex ellipsoid in $\mathbb{C}^{n}$

$$
D=\left\{z \in \mathbb{C}^{n} \mid \rho(z)<0\right\}
$$

where

$$
\rho(z)=\sum_{j=1}^{n}\left\|z_{j}\right\|^{2 m_{j}}-1, \quad m_{j} \geq 1, \quad n \geq 2 .
$$

Denote by $\mathcal{C}_{(0, q)}^{k}(\bar{D})$ the space of all continuous $(0, q)$-forms on $D$ and having continuous derivatives up to $[k]$ on $D$ satisfying $H^{\prime}$ older condition of order $k-[k]$. The corresponding norm is denoted by $\|\cdot\|_{k, D}$. The following result has been proved in [15].

Theorem 3. Let $D=\left\{z \in \mathbb{C}^{n} \mid \rho(z)<0\right\}$ be a complex ellipsoid in $\mathbb{C}^{n}$ defined as above. Let $m=\max \left\{2 m_{j}, j=1, \ldots, n\right\}$. Then there are linear solution operators $S_{q}: \mathcal{C}_{0, q}^{1}(\bar{D}) \longrightarrow \mathcal{C}_{0, q-1}^{1}(\bar{D})$ such that $\bar{\partial} S_{q} f=f$ for every $\bar{\partial}$-closed form $f \in \mathcal{C}_{0, q}(\bar{D}) \cap \mathcal{C}_{0, q}^{1}(D), 1 \leq q \leq n$. If in addition $f \in \mathcal{C}_{0, q}(\bar{D}) \cap \mathcal{C}_{0, q}^{k}(D)$, $k \geq 1,0<\epsilon<\frac{1}{m}$, then there exists a constant $C_{k, \epsilon}>0$ such that

$$
\left\|S_{q} f\right\|_{k+\epsilon, D} \leq C_{k, \epsilon}\|f\|_{k, D}
$$

This theorem enables us to prove our second result on $\mathcal{C}^{k}$-holomorphic approximation on complex ellipsoids.

Theorem 4. Let $D$ be the complex ellipsoid given as in Theorem 3. Then for every function $f$ in $\mathcal{C}^{k}(\bar{D}) \cap \mathcal{O}(D)$, $k \geq 1$, there is a sequence of functions $f_{m}$ in $\mathcal{O}(\bar{D})$ such that $\lim _{m \rightarrow \infty}\left\|f_{m}-f\right\|_{\mathcal{C}^{k}(\bar{D})}=0$.

Our approach in the proofs of Theorems 2 and 4 is to cover $\partial D$ by finitely many open charts, approximate the function in $\mathcal{C}(\bar{D}) \cap \mathcal{O}(D)$ by a holomorphic function in each of the charts and patch these local approximations into a global one by solving an additive Cousin problem with bounds for locally defined forms or functions via estimates for $\bar{\partial}$ in the corresponding $L^{p}$ or $\mathcal{C}^{k}$ settings.

For any two expressions $A$ and $B$ which depending on several parameter, we will use $A \lesssim B$ to mean that there is a constant $C$ independent of the parameters under consideration such that $A \leq C B$. Since the proof of Theorem 4 will be very similar to that of Theorem 2 then we will present a detailed proof for Theorem 2 only. 


\section{Additive Cousin Problem}

In this section we solve the additive Cousin problem with $L^{p}$-bounds.

Theorem 5. Let $D_{\phi}$ be as in Theorem 1, let $\left\{U_{j}\right\}_{j=0}^{N}$ be an open covering of $\overline{D_{\phi}}$ and set $V_{j}=U_{j} \cap D_{\phi}$. If $h_{i j} \in \mathcal{O}\left(V_{i} \cap V_{j}\right)$ are Cousin data such that

$$
h_{i j}+h_{j i}=0 ; \quad h_{i j}+h_{j k}+h_{k i}=0 \quad \forall i, j, k=0,1, \ldots, N .
$$

Then there are functions $h_{j} \in \mathcal{O}\left(V_{j}\right), 0 \leq j \leq N$, such that

$$
h_{i j}=h_{j}-h_{i} \text { on } V_{i} \cap V_{j} .
$$

Moreover, there is a finite constant $C>0$ satisfying the estimates

$$
\left\|h_{j}\right\|_{L^{p}\left(V_{j}\right)} \leq C M_{p}, \quad 1 \leq p \leq \infty,
$$

where $M_{p}=M_{p}\left(\left\{h_{i j}\right\}\right)=\max \left\{\left\|h_{i j}\right\|_{L^{p}\left(V_{i} \cap V_{j}\right)} ; i, j=0,1, \ldots, N\right\}$.

Proof. Let $\left\{\theta_{j}\right\}_{j=0}^{N}$ be a partition of unity subordinate to the covering $\left\{U_{j}\right\}$ so that $\theta_{j} \in \mathcal{C}_{0}^{\infty}\left(U_{j}\right)$ and $\sum_{j=0}^{N} \theta_{j}=1$ in a neighborhood of $\overline{D_{\phi}}$. Since $\left\{V_{j}\right\}$ is locally finite, the sum $\sum_{v=0}^{N} \theta_{v} h_{v j}$ defines a $\mathcal{C}^{\infty}$-function $\beta_{j}$ on each $V_{j}$ and by Minkowski's inequality, we have that

$$
\left\|\beta_{j}\right\|_{L^{p}\left(V_{J}\right)} \lesssim M_{p}
$$

Further, in view of (1) and using $\sum_{v=0}^{N} \theta_{v}=1$, we have that

$$
\beta_{j}-\beta_{i}=\sum_{v=0}^{N} \theta_{v}\left(h_{v j}-h_{v i}\right)=\sum_{v=0}^{N} \theta_{v} h_{i j}=h_{i j}
$$

This together with $\bar{\partial} h_{i j}=0$ imply that

$$
\bar{\partial} \beta_{j}=\bar{\partial} \beta_{i} \quad \text { on } V_{i} \cap V_{j} \quad \forall i, j=0,1, \ldots, N .
$$

By patching together the locally defined smooth $(0,1)$-forms $\bar{\partial} \beta_{j}$ we obtain a global smooth $(0,1)$-form $f$ on $D_{\phi}$ with $\bar{\partial} \beta_{j}=f$ on $V_{j}$ for each $j=0,1, \ldots, N$.

It is clear that $\bar{\partial} f=0$ on $D_{\phi}$. Now we estimate $f$ in the $L^{p}\left(D_{\phi}\right)$-norm, since $\bar{\partial} \beta_{j}=\sum_{v=0}^{N} \bar{\partial} \theta_{j} \cdot h_{v j}$, the estimate (3) gives

$$
\|f\|_{L_{0,1}^{p}\left(D_{\phi}\right)} \leq \sum_{j=0}^{N}\left\|\bar{\partial} \beta_{j}\right\|_{L_{0,1}^{p}\left(\left(V_{j}\right)\right.} \lesssim M_{p}<\infty .
$$

Then, by Theorem 1 , there is a function $u$ in $L^{p}\left(D_{\phi}\right)$ such that $\bar{\partial} u=f$ in $D_{\phi}$ and

$$
\|u\|_{L^{p}\left(D_{\phi}\right)} \leq C\|f\|_{L_{0,1}^{p}\left(D_{\phi}\right)} \leq C^{\prime} M_{p}, 1 \leq p \leq \infty .
$$

Set

$$
h_{j}=\beta_{j}-u \quad \text { on } V_{j} .
$$

Then $h_{j}$ is holomorphic on $V_{j}$ for each $j$ and by (4), we further have

$$
h_{j}-h_{i}=\left(\beta_{j}-u\right)-\left(\beta_{i}-u\right)=h_{i j} \quad \text { on } V_{i} \cap V_{j} \quad \forall i, j=0,1, \ldots, N .
$$


Finally, the estimates (3) and (5) imply (2). This completes the proof.

\section{Proof of Theorem 2}

The proof follows by using arguments from [16]. Cover $\partial D_{\phi}$ by finitely many neighborhoods $U_{1}, U_{2}, \ldots, U_{N}$ of boundary points $p_{j}$ such that for each $U_{j}$ there is a constant $\tau_{0}>0$ so that if $n_{j}$ is the inner unit normal to $\partial D_{\phi}$ at $p_{j}$, then $z+\tau n_{j} \in D_{\phi}$ for all $z \in \overline{D_{\phi}} \cap U_{j}$ and $0<\tau<\tau_{0}$.

For $0<\tau<\tau_{0}$, we set

$$
U_{0}^{\tau}=D_{\phi}, \quad U_{j}^{\tau}=\left\{z=w-\tau n_{n} ; w \in D_{\phi} \cap U_{j}\right\} \cap U_{j} \text { for } j=1,2, \ldots, N .
$$

The family of open sets $\left\{U_{j}^{\tau}\right\}_{j=0}^{N}$ forms an open cover of $\overline{D_{\phi}}$ provided that $\tau_{0}$ is chosen sufficiently small. We then have the following local approximating Lemma.

Lemma 1. Let $f \in H^{p}\left(D_{\phi}\right), 1 \leq p \leq \infty$. For $0<\tau<\tau_{0}$ define $f_{0}^{\tau}=f$ and $f_{j}^{\tau}=f\left(z+n_{j} \tau\right)$ for $1 \leq j \leq N$. Then we have the following statements:

(A) $f_{j}^{\tau} \in H^{p}\left(U_{j}^{\tau}\right)$ for $j=0, \ldots, N$,

(B) $\lim _{\tau \rightarrow 0} f_{j}^{\tau}=f$ pointwise on $D_{\phi} \cap U_{j}$,

(C) If $p<\infty$ or $p=\infty$ and $f$ is in $\mathcal{C}\left(\bar{D}_{\phi}\right) \cap \mathcal{O}\left(D_{\phi}\right)$

$$
\lim _{\tau \rightarrow 0}\left\|f-f_{j}^{\tau}\right\|_{L^{p}\left(D_{\phi} \cap U_{j}\right)}=0 .
$$

Proof. Statements (A) and (B) follow obviously from the definitions of $U_{j}^{\tau}$ and $f_{j}^{\tau}$. For $p<\infty$, Equation (6) follows from the fact that translation defines a continuous operator on $L^{p}$-spaces. For $p=\infty$, it follows from the uniform continuity of $f$.

Unfortunately, those local approximating functions $f_{j}^{\tau}$ do not match up together to form a global holomorphic function on the closure of $D$, because

$$
h_{i j}^{\tau}=f_{j}^{\tau}-f_{i}^{\tau} \text { on } U_{i}^{\tau} \cap U_{j}^{\tau}
$$

will in general be different from zero if $i \neq j$. However, we have

Corollary 1. Define

$$
h_{i j}^{\tau}=f_{j}^{\tau}-f_{i}^{\tau} \text { on } U_{i}^{\tau} \cap U_{j}^{\tau},
$$

and

$$
M_{p}^{\tau}\left(\left\{h_{i j}^{\tau}\right\}\right)=\max _{0 \leq i, j \leq N}\left\|h_{i j}^{\tau}\right\|_{L^{p}\left(U_{i}^{\tau} \cap U_{j}^{\tau}\right)}
$$

Then

$$
\lim _{\tau \rightarrow 0} M_{p}^{\tau}\left(\left\{h_{i j}^{\tau}\right\}\right)=0 \quad \text { if } p<\infty \text { or } p=\infty \text { and } f \in \mathcal{C}\left(\bar{D}_{\phi}\right) \cap \mathcal{O}\left(D_{\phi}\right),
$$

and in addition, if $f \in L^{\infty}\left(D_{\phi}\right)$, we have

$$
M_{\infty}^{\tau}\left(\left\{h_{i j}^{\tau}\right\}\right) \lesssim\|f\|_{L^{\infty}\left(D_{\phi}\right)}
$$

Proof. The inequality (9) is obvious. For (8), fix $i$ and $j$ and set $W=U_{i} \cap U_{j} \cap D_{\phi}$, then

$$
\left\|h_{i j}^{\tau}\right\|_{L^{p}(W)} \leq\left\|f_{j}^{\tau}-f\right\|_{L^{p}(W)}+\left\|f_{i}^{\tau}-f\right\|_{L^{p}(W)}
$$

and by (6), we obtain

$$
\left\|h_{i j}^{\tau}\right\|_{L^{p}(W)} \rightarrow 0 \text { as } \tau \rightarrow 0,
$$


since $\operatorname{Vol}\left(U_{i}^{\tau} \cap U_{j}^{\tau} \cap \backslash W\right) \rightarrow 0$ as $\tau \rightarrow 0$. This implies (8) for $p<\infty$. The case when $p=\infty$ follows also from the uniform continuity of $f$.

End Proof of Theorem 2

Choose $\theta_{j} \in \mathcal{C}_{0}^{\infty}\left(U_{0}^{\tau}\right)$ and $\theta_{0} \in \mathcal{C}_{0}^{\infty}\left(D_{\phi}\right)$ so that $\sum_{j=0}^{N} \theta_{j}=1$ on a neighborhood $W$ of $\overline{D_{\phi}}$. For each $\tau<\tau_{0}$ we choose $\eta(\tau)$ such that

$$
D_{\phi}^{\eta(\tau)}=\left\{z \in \mathbb{C}^{n} \mid \sigma_{\eta(\tau)}(z)=\sigma(z)-\eta(\tau)<0\right\} \subset W \cap \bigcup_{j=0}^{N} U_{j}^{\tau} .
$$

It is obvious that $\eta(\tau) \rightarrow 0$ as $\tau \rightarrow 0$. By choosing $\tau_{0}$ small enough, we can achieve that

$$
\sup \theta_{j} \cap \bar{D}_{\phi}^{\eta(\tau)} \subset U_{j}^{\tau} \quad \text { for all } 0<\tau<\tau_{0} \text { and } j=0, \ldots, N .
$$

If $\tau_{0}$ is sufficiently small, we see moreover that the integral kernels $K_{q}^{\eta(\tau)}(\xi, z)$ are well-defined for $0<\tau<\tau_{0}$, and so there are $\bar{\partial}$-solving linear operators $T_{q}^{\eta(\tau)}: L_{0, q}^{1}\left(D_{\phi}^{\eta(\tau)}\right) \rightarrow L_{0, q-1}^{1}\left(D_{\phi}^{\eta(\tau)}\right)$. Since the derivatives of the defining function $\sigma_{\eta(\tau)}$ of $D_{\phi}^{\eta(\tau)}$ are independent of $\tau$ and the volume of $D_{\phi}^{\eta(\tau)}$ is bounded by a constant independent of $\tau$ for $0<\tau<\tau_{0}$, then the $L^{p}$-estimates given by Theorem 1 for the operators $T_{q}^{\eta(\tau)}$ are independent of $\tau$ for $0<\tau<\tau_{0}$.

Set $V_{j}^{\tau}=D_{\phi}^{\eta \tau} \cap U_{j}^{\tau}$ and apply Theorem 5 to the functions $h_{i j}^{\tau} \in \mathcal{O}\left(V_{i}^{\tau} \cap V_{j}^{\tau}\right)$, we get functions $h_{j}^{\tau} \in \mathcal{O}\left(V_{j}^{\tau}\right)$ such that

$$
h_{i j}^{\tau}=h_{j}^{\tau}-h_{i}^{\tau} \text { on } V_{i}^{\tau} \cap V_{j}^{\tau}
$$

and there is a constant $C>0$, independent of $\tau$, satisfying the estimate

$$
\left\|h_{j}^{\tau}\right\|_{L^{p}\left(V_{j}^{\tau}\right)} \leq C M_{p}^{\tau}\left(\left\{h_{i j}^{\tau}\right\}\right), \quad 1 \leq p \leq \infty .
$$

It follows from (7) and (10) that

$$
f_{j}^{\tau}-h_{j}^{\tau}=f_{i}^{\tau}-h_{i}^{\tau} \text { on } V_{i}^{\tau} \cap V_{j}^{\tau}
$$

Hence $\left\{f_{j}^{\tau}-h_{j}^{\tau}\right\}_{j=0}^{N}$ defines a holomorphic function $f_{j}^{\tau}$ on $D_{\phi}^{\eta(\tau)}$ so that

$$
f^{\tau}=f_{j}^{\tau}-h_{j}^{\tau} \quad \text { on } V_{j}^{\tau} .
$$

We finally estimate the $L^{p}$-norm of $f-f^{\tau}$ as $\tau \rightarrow 0$. Since $f_{0}^{\tau}=f$, it follows from (12) and (11) that

$$
\left\|f-f^{\tau}\right\|_{L^{p}\left(D_{\phi}\right)} \leq \sum_{j=0}^{N}\left\|f-f_{j}^{\tau}\right\|_{L^{p}\left(D_{\phi} \cap U_{j}\right)}+C(N+1) M_{j}^{\tau}\left(h_{i j}^{\tau}\right) .
$$

If $p<\infty$ either or $p=\infty$, from this last inequality together with (6) and (8), we see that

$$
\left\|f-f^{\tau}\right\|_{L^{p}\left(D_{\phi}\right)} \rightarrow 0 \quad \text { as } \tau \rightarrow 0
$$

for all $f \in \mathcal{C}\left(\bar{D}_{\phi}\right) \cap \mathcal{O}\left(D_{\phi}\right)$.

For the case when $f \in H^{\infty}\left(D_{\phi}\right)$, using again (12) and (11) together with (9), we get

$$
\left\|f^{\tau}\right\|_{L^{\infty}\left(D_{\phi}\right)} \leq C\|f\|_{L^{\infty}\left(D_{\phi}\right)} \quad \forall 0<\tau<\tau_{0},
$$


where the constant $C$ is independent of $\tau$. Since $H^{\infty}\left(D_{\phi}\right) \subset H^{p}\left(D_{\phi}\right)$ for all $p<\infty$, then (13) still holds true for any $p<\infty$. This completes the proof of Theorem 2 .

Conflicts of Interest: The author declares no conflict of interest.

\section{References}

1. Chalendar, I.; Leblond, J; Partington, J.R. Approximation problems in some holomorphic spaces, with applications. In Systems, Approximation, Singular Integral Operators, and Related Topics. Operator Theory: Advances and Applications; Borichev, A.A., Nikolski, N.K., Eds.; Birkhäuser: Basel, Switzerland, 2001; Volume 129; pp. 143-168.

2. Mergelyan, S.N. Uniform approximation to functions of a complex variable. Uspehi Mat. Nauk 1952, 7, 31-122.

3. Henkin, G.M. Integral representation of functions which are holomorphic in strictly pseudoconvex regions, and some applications. Math. USSR-Sb. 1969, 7, 597-616. [CrossRef]

4. Lieb, I. Ein Approximationssatz auf streng pseudokonvexen Gebieten. Math. Ann. 1969, 184, 56-60. [CrossRef]

5. Kerzman, N. Hölder and $L^{p}$-estimates for solutions of $\bar{\partial} u=f$ in strongly pseudoconvex domains. Comm. Pure Appl. Math. 1971, 24, 301-379. [CrossRef]

6. Fornæss, J.E. Embedding strictly pseudoconvex domains in convex domains. Am. J. Math. 1976, 98, 529-569. [CrossRef]

7. Ahem, P.; Schneider, R. Holomorphic Lipschitz functions in pseudoconvex domains. Ann. Math. 1978, $101,543-565$.

8. Lieb, I.; Range, R.M. Ein Lösungsoperator für den Cauchy-Riemann Komplex mit $C^{k}$-Abschatzungen. Math. Ann. 1980, 253, 145-164. [CrossRef]

9. Lieb, I.; Michel, J. The Cauchy-Riemann Complex, Integral Formulae and Neumann Problem; Aspects of Mathematics, E 34; Friedr. Vieweg \& Sohn: Braunschweig, Germany, 2002.

10. Fornæss, J.E.; Nagel, A. The Mergelyan property for weakly pseudoconvex domains. Manuscripta Math. 1977, 22, 199-208. [CrossRef]

11. Cho, S. On the Mergelyan Approximation Property on Pseudoconvex Domains in $\mathbb{C}^{n}$. Proc. Am. Math. Soc. 1998, 26, 2285-2289 [CrossRef]

12. Ha, L.K. $L^{p}$-approximation of holomorphic functions on a class of convex domains. Bull. Aust. Math. Soc. 2018, 97, 446-452. [CrossRef]

13. Diederich, K.; Fornæss, J.E. A strange bounded smooth domain of holomorphy. Bull. Am. Math. Soc. 1976, 182, 74-76. [CrossRef]

14. Adachi, K.; Cho, H.R. $L^{p}(1 \leq p \leq \infty)$ estimates for $\bar{\partial}$ on a certain pseudoconvex domain in $\mathbb{C}^{n}$. Nagoya Math. J. 1997, 148, 127-136. [CrossRef]

15. Ryczaj, J. $\mathcal{C}^{k}$-estimates for the Cauchy-Riemann equations on certain weakly pseudoconvex domains. Colloq. Math. 1987, 52, 298-304. [CrossRef]

16. Range, R.M. Holomorphic Functions and Integral Representations in Several Complex Variables; Springer: New York, NY, USA, 1986.

(C) 2019 by the author. Licensee MDPI, Basel, Switzerland. This article is an open access article distributed under the terms and conditions of the Creative Commons Attribution (CC BY) license (http://creativecommons.org/licenses/by/4.0/). 